\title{
Author Correction: Melatonin and mitochondrial function during ischemia/reperfusion injury
}

\author{
Zhiqiang Ma ${ }^{1,2} \cdot$ Zhenlong Xin ${ }^{3} \cdot$ Wencheng $\mathrm{Di}^{4} \cdot$ Xiaolong $\mathrm{Yan}^{2} \cdot{\text { Xiaofei } \mathrm{Li}^{2} \cdot \text { Russel J. Reiter }}^{5} \cdot$ Yang Yang ${ }^{1,3}$
}

Published online: 24 April 2018

(c) Springer International Publishing AG, part of Springer Nature 2018

\section{Author Correction: Cell. Mol. Life Sci. (2017) 74:3989-3998 https://doi.org/10.1007/s00018-017-2618-6}

In the original publication, affiliations were incorrectly published for the authors Russel J. Reiter and Yang Yang. The correct affiliations should read as follows.

Russel J. Reiter

5. Department of Cellular and Structural Biology, UT Health Science Center, 7703 Floyd Curl Drive, San Antonio, TX 78229, USA

Yang Yang

1. Key Laboratory of Resource Biology and Biotechnology in Western China, Ministry of Education, Faculty of Life Sciences, Northwest University, 229 Taibai North Road, Xi' an 710069, China

The original article can be found online at https://doi.org/10.1007/ s00018-017-2618-6.

Russel J. Reiter

reiter@uthscsa.edu

$\triangle$ Yang Yang

yang200214yy@163.com

1 Key Laboratory of Resource Biology and Biotechnology in Western China, Ministry of Education, Faculty of Life Sciences, UT Health San Antonio, 229 Taibai North Road, Xi' an 710069, China

2 Department of Thoracic Surgery, Tangdu Hospital, Fourth Military Medical University, 1 Xinsi Road, Xi' an 710038, China

3 Department of Biomedical Engineering, Fourth Military Medical University, 169 Changle West Road, Xi'an 710032, China

4 Department of Cardiology, Affiliated Drum Tower Hospital, Nanjing University Medical School, 321 Zhongshan Road, Nanjing 210008, Jiangsu, China

5 Department of Cellular and Structural Biology, UT Health Science Center, 7703 Floyd Curl Drive, San Antonio, TX 78229, USA
3. Department of Biomedical Engineering, Fourth Military Medical University, 169 Changle West Road, Xi'an 710032, China 